\title{
RECOMENDAÇÕES PARA CALIBRAÇÃo EM QUÍMICA ANALÍTICA - PARTE I. FUNDAMENTOS E CALIBRAÇÃO COM UM COMPONENTE (CALIBRAÇÃO UNIVARIADA) ${ }^{*}$
}

\section{Benício de Barros Neto}

Departamento de Química Fundamental, Universidade Federal de Pernambuco, Av. Prof. Luis Freire, s/n, 50740-540 Recife - PE

Maria Fernanda Pimentel

Departamento de Engenharia Química, Universidade Federal de Pernambuco, Rua Teresa Méllia, s/n, 50740-521 Recife - PE

Mário César Ugulino Araújo*

Departamento de Química, Universidade Federal da Paraíba - Campus I, CP 5093, 58059-900 João Pessoa - PB

Recebido em 13/12/01

\begin{abstract}
This paper is a translation from IUPAC nomenclature document by K. Danzer and L. A. Currie (Pure Appl. Chem., 1998, 70(4), 993-1014). Its goal is to establish an uniform and meaningful approach to terminology (in Portuguese), notation, and formulation for calibation in analytical chemistry. In this first part, general fundamentals of calibration are presented, namely for both relationships of qualitative and quantitative variables (relations between variables characterizing certain types analytes of the measured function on the other hand and between variables characterizing the amount or concentration of the chemical species and the intensities of the measured signals, on the other hand). On this basis, the fundamentals of the common single component calibration (Univariate Calibration) which models the relationship $y=f(x)$ between the signal intensities $y$ and the amounts or concentrations $x$ of the analyte under given conditions are represented. Additional papers will be prepared dealing with extensive relationships between several intensities and analyte contents, namely with multivariate calibrations and with optimization and experimental design.
\end{abstract}

\section{INTRODUÇÃO}

A calibração, em geral, é uma operação que relaciona uma grandeza de saída com uma grandeza de entrada, para um sistema de medida sob determinadas condições.

No processo de medição química (PMQ) ${ }^{1}$, as grandezas de entrada são grandezas analíticas que caracterizam os tipos de analitos (espécies químicas), $q_{i}$, e suas quantidades ou concentrações, $x$. As grandezas de saída são representadas por valores medidos, isto é, sinais observados em posições $z_{j}$ com intensidades $y_{z j^{j}} \mathrm{O}$ caso mais comum, no qual se determina uma relação entre a quantidade ou concentração (conteúdo) de um certo analito, $x_{q i}$, e a intensidade de um sinal, $y_{z j}$, é apenas um caso especial de calibração.

Em um sentido mais amplo, a calibração em Química Analítica se refere à relação entre uma função analítica, $x=f(q)$, que representa um perfil de distribuição de analitos e suas quantidades ou con- centrações em uma dada amostra-teste (Figura 1, lado esquerdo), e uma função medida $y=g(z)$, que pode ser representada por um espectro, um cromatograma, etc. (Figura 1, lado direito) ${ }^{2}$.

Existem portanto quatro grandezas, $q, x, z$ e $y$, que precisam ser relacionadas entre si, numa situação que pode ser caracterizada pelo diagrama pseudo-tetradimensional da Figura 2. A relação entre a espécie e seu sinal característico aparece em primeiro plano. Por trás dela, a relação entre o sinal e a concentração é estabelecida. Juntas, estas duas relações estabelecem a composição da amostra. Estas relações gerais se refletem em três aplicações analíticas relevantes:

1. Calibração q-z: Calibração de grandezas que caracterizam posições típicas de sinal, $z$, para identificação de componentes e análise qualitativa:

$$
z=f(q)+e_{z} \approx L \cdot q+e_{z}
$$

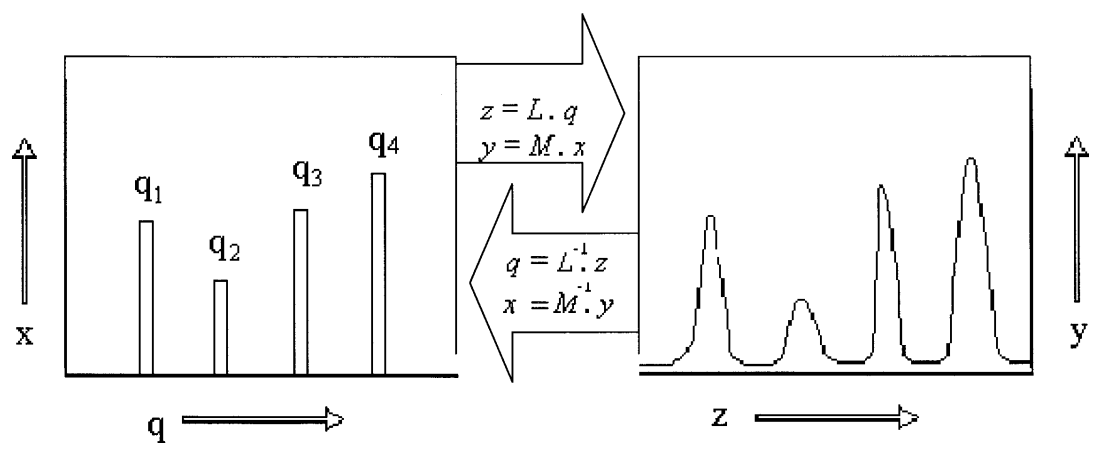

Figura 1. Relação entre a função analítica $x=f(q)$ e a função medida $y=g(z)$

*e-mail: laqa@quimica.ufpb.br

\# O presente artigo é uma tradução para o português (aprovada pelo Comitê Brasileiro para Assuntos de Química junto à IUPAC), do artigo

"Guidelines for Calibration in Analytical Chemistry. Part 1. Fundamentals and Single Component Calibration" publicado em Pure Appl. Chem. 1998,

70, 993, preparado por K. Danzer e L. A. Currie. 
onde $f$ representa a relação funcional entre $z$ e $q, e_{z}$ representa o erro de medição em $z$ e $L$ é o operador (aproximadamente) linear que transforma $q_{i}$ (que é uma grandeza específica do componente, como número atômico, número de massa, ou um valor de energia típico) em $z$.

2. Calibração $y-x$ : Calibração de grandezas que caracterizam a intensidade de uma resposta observada $y$ para um dado analito $q_{i}$, em análises quantitativas de um componente:

$$
y=F(x)+e_{y} \approx M \cdot x+e_{y}
$$

onde $F$ é a relação funcional entre $y$ e $x$ (a função de calibração, em sentido mais estrito), $e_{y}$ representa o erro de medição em $y$, e $M$ é o operador aproximadamente linear que transforma $x$ em $y$.

\section{Calibração quantitativa multicomponente:}

$$
\mathbf{Y}=\mathbf{A} \cdot \mathbf{X}+\mathbf{E}
$$

onde $\mathrm{Y}$ representa a matriz dos valores medidos, $\mathrm{X}$ é a matriz das concentrações ou quantidades dos analitos, A é a matriz de sensibilidade que transforma $\mathrm{X}$ em $\mathrm{Y}$, e E é a matriz dos erros. A calibração quantitativa multicomponente, que será o tema de um segundo artigo, é feita através de técnicas de regressão múltipla ou multivariada.

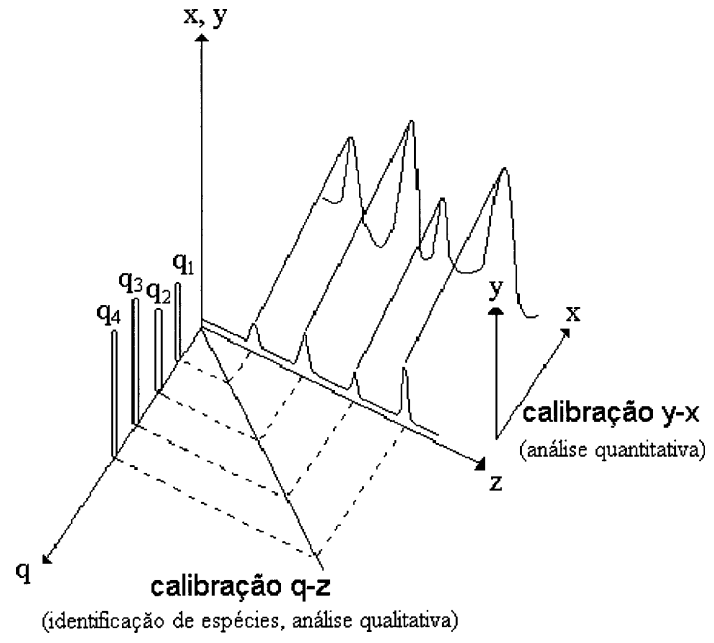

Figura 2. Representação pseudo-tetradimensional da ligação entre a calibração qualitativa e a calibração quantitativa. A relação q-z corresponde a uma função determinística, $(i)$. Freqüentemente existem relações empíricas, como (ii) e (iii)

\section{FUNDAMENTOS}

Calibração em Química Analítica é a operação que determina a relação funcional entre valores medidos (intensidades $y$ de sinais em certas posições $z_{j}$ ) e grandezas analíticas caracterizando os tipos de analitos $q_{i}$ e suas quantidades ou concentrações $x$. A calibração inclui a seleção do modelo (isto é, a especificação da sua forma funcional), a estimativa dos parâmetros do modelo e dos erros associados a essas estimativas, e a validação do modelo.

Calibração para identificação de espécies e análise qualitativa (calibração $q-z$, ou, mais especificamente, calibração de parâmetros

${ }^{a}$ Evaluation. (N.T.) analíticos que caracterizam os tipos de espécies químicas) é o ajuste de um modelo (estimativa e validação de seus parâmetros) para a relação entre $z$ e $q$, para fins de identificação e análise qualitativa usando-se a Eq. (1).

$z=f(q)+e_{z} \approx L \cdot q+e_{z}$

Na prática analítica, a calibração $q-z$ refere-se à localização de sinais em uma configuração característica da espécie presente, em escalas de energia ou proporcionais à energia, como comprimentos de onda, freqüências ou razões massa/carga em espectrômetros de massas, ou o tempo em cromatogramas.

Na identificação e na análise qualitativa, o operador linear $L$ pode $\operatorname{ser}^{2}$ :

i. uma função determinística baseada em leis naturais, $z=f_{\text {det }}(q)$, como a lei de Moseley sobre a dependência das frequiências de raios-X em relação ao número atômico ${ }^{3}$, ou

ii. uma função empírica, $z=f_{\text {emp }}(q)$, como os índices de Kovats para normalização de tempos de retenção com base em uma série homóloga de referência, em cromatografia gasosa ${ }^{4}$, ou

iii. uma relação empírica, $z=\operatorname{emp}(q)$, representada por tabelas e atlas, como a tabela de Colthup para vibrações características ${ }^{5}$, os atlas do ferro em espectroscopia de emissão atômica ${ }^{6}$ e outros tipos de tabelas de comprimentos de onda?

Enquanto que as relações $z=f_{\text {det }}(q)$ são conhecidas com base em leis naturais, as funções empíricas $z=f_{e m p}(q)$ para identificação e análise qualitativa geralmente são estimadas através de um ajuste por mínimos quadrados (linear) de valores $z$ observados para uma série de padrões dos componentes puros ou para um padrão multicomponente. Calibrações por meio de relações empíricas $z=$ $\operatorname{emp}(q)$ dadas por tabelas, atlas e gráficos são obtidas através da classificação dos resultados experimentais.

Calibração para análise quantitativa é a determinação da relação funcional entre $y$ e $x$, da forma:

$y=F(x)+e_{y}$

onde $F$ é a função de calibração.

Na maioria dos casos, a função de calibração precisa considerar a influência de todos os constituintes e interferentes relevantes sobre a resposta. Portanto, $y$ depende de um vetor $\mathbf{x}=\left(x_{a}, x_{b} \ldots x_{m}, x_{n} \ldots x_{q}\right)$ que contém as quantidades ou concentrações do analito de interesse, $x_{a}$, dos componentes que o acompanham, $\left(x_{b} \ldots x_{m}\right)$, e dos fatores interferentes, $\left(x_{n} \ldots x_{q}\right)$ :

$y=F(\mathbf{x})+e_{y}$

Nas circunstâncias mais desejáveis, a Eq. (4) é uma equação vetorial linear. O emprego da Eq. (4) para o ajuste de modelos é assunto para o planejamento experimental e a otimização, que serão abordados em um terceiro artigo.

Na calibração mais simples, de acordo com a Eq. (2), para uma dada quantidade ou concentração de $x=x_{a}$ onde não seja preciso considerar outros componentes e fatores interferentes, $y$ é uma grandeza escalar. No caso mais geral, $y$ pode ser uma função da variável $z$. A relação básica assume então a forma:

$y(z)=M(z) x+e_{y}$

e passa a representar um perfil de distribuição característico, como um espectro de um componente puro.

\section{Função de avaliação ${ }^{1,8}$}

Em geral, a função de avaliação ${ }^{\text {a }}$ (função analítica) é o inverso da função de calibração da Eq. 2: 
$x=F^{-1}(y)$

desde que a relação entre o valor medido $y$ e a concentração ou quantidade do analito $x$ tenha sido obtida por calibração, que é o usual em Química Analítica. Entretanto, dependendo na natureza do método analítico, existem outros tipos de procedimento de avaliação, como os baseados em leis naturais. A determinação das quantidades ou concentrações dos analitos pode ser baseada em medidas absolutas, medidas relativas ou medidas de referência ${ }^{9}$.

Medidas absolutas, medidas definidas e medidas de referência são baseadas em equações do tipo geral:

$y=A \cdot x$

onde a sensibilidade $A$ é geralmente definida como a derivada $d y / d x$. No caso de modelos lineares, $A$ é dado por $\Delta y / \Delta x^{1}$. Para esses três tipos de medidas analíticas, a sensibilidade é dada por relações matemáticas bem definidas, a saber:

(a) Medidas absolutas: Por grandezas fundamentais, como a constante de Faraday e razões entre as massas atômicas e molares;

(b) Medidas definidas: Por grandezas fundamentais combinadas com constantes empíricas conhecidas e transferíveis (por exemplo, coeficiente de absorção molar, condutividade a uma diluição definida, coeficientes de difusão para um determinado meio), e algumas vezes complementadas por um fator empírico (título, por exemplo);

(c) Medidas de referência direta: Pela relação entre o valor medido e o teor ou concentração de um material de referência $(\mathrm{R})$ :

$A=y_{R} / x_{R}$

Medidas absolutas não necessitam de calibração permanente ${ }^{\mathrm{b}}$. Medidas definidas ou medidas de referência direta precisam apenas de uma medida para comparação (como um padrão titulométrico) ou de uma medida de referência (material de referência ou amostra fortificada, isto é, contendo uma quantidade adicionada conhecida ${ }^{c}$ ) Medidas de referência indiretas se baseiam em funções de calibração empíricas, que são freqüentemente descritas por modelos lineares:

$y=B+A x+e_{y}$

onde a interseção $B$ corresponde ao branco experimental e o coeficiente angular $A$ corresponde à sensibilidade experimental. Os parâmetros $A$ e $B$ normalmente são estimados usando regressão linear com ajuste por mínimos quadrados ${ }^{\mathrm{d}}$. O termo $e_{y}$ representa o erro nas medidas de $y$. Na prática analítica, alguns métodos que em princípio usam medidas definidas (como espectrofotometria ou polarografia) também são calibrados por mínimos quadrados, para que sejam obtidas estimativas confiáveis de $A$.

Funções de calibração correspondendo à Eq. (8) geralmente não são transferíveis ao longo do tempo, nem de um laboratório para outro. Entretanto, no caso de relações sem branco ou corrigidas em relação ao branco, dadas por
$y=A x+e_{y}$

os métodos podem ser calibrados robustamente, sob condições experimentais fixas. Sob condições operacionais padronizadas, os coeficientes de sensibilidade experimentais (fatores de sensibilidade) são transferíveis ao longo do tempo e entre laboratórios. Por causa desta transferibilidade, tais métodos às vezes são denominados de métodos "isento de padrão" . Métodos deste tipo têm sido desenvolvidos, por exemplo, em espectrografia de emissão ${ }^{10}$, em espectroscopia de massas com fonte de centelha ${ }^{11}$, e em espectroscopia de fluorescência de raios- $\mathrm{X}^{12}$, para análise multielementar semiquantitativa. Deve-se distinguir os métodos "isento de padrão" dos métodos sem calibração. Estes últimos usam medidas absolutas, como já foi mencionado 9 .

\section{CALIBRAÇÃO POR MÍNIMOS QUADRADOS}

\section{Modelo linear de calibração}

Admitindo-se que os erros nas medidas têm média zero e não são correlacionados, uma função linear (Eq. 8) pode ser ajustada aos valores experimentais usando-se a estimativa por mínimos quadra$\underline{\text { dos }}(\mathrm{MQ})$, também chamada de estimativa por mínimos quadrados ordinários (MQO).

2. Com as relações fundamentais

- Modelo : $y_{i}=B+A x_{i}+e_{y i}=E\left(y_{i}\right)+e_{y i}$

- Estimativa: $\hat{y}_{i}=\hat{B}+\hat{A} x_{i}$

- Resíduo: $d_{y i}=y_{i}-\hat{y}_{i}=y_{i}-\hat{B}-\hat{A} x_{i}$

onde $E\left(y_{i}\right)$ é o valor esperado de $y_{i}$, o critério geral dos mínimos quadrados é expresso pela soma quadrática residual ${ }^{\mathrm{f}}, S Q_{r}{ }^{13}$, dada por

$$
S Q_{r}=\sum_{i=1}^{m}\left[\left(y_{i}-\hat{y}_{i}\right) / \sigma_{i}\right)^{2}=\sum_{i=1}^{m}\left(d_{y i} / \sigma_{i}\right)^{2}
$$

onde $\sigma_{i}$ é o desvio padrão no ponto $i$ e $m$ é o número de medidas da calibração [V. Eq. (20)].

Nota 1: Um critério correspondente pode ser formulado para a determinação de $x$ a partir de $y$ (admitido sem erro) pela estimativa $\hat{x}=\hat{B}_{y}+\hat{A}_{y} y$, mas este modelo geralmente não é relevante para a calibração analítica.

A $S Q_{r}$ deve ser minimizada de acordo com a Equação (12):

$$
\sum_{i=1}^{m}\left(d_{y i} / \sigma_{i}\right)^{2} \stackrel{!}{=} \min
$$

O símbolo $\stackrel{!}{=}$ significa que a expressão no lado esquerdo tem de assumir seu valor mínimo. Dependendo do comportamento dos erros, o critério dos mínimos quadrados deve ser modificado, como está especificado a seguir.

\footnotetext{
${ }^{\mathrm{b}}$ Exceto pelo fato de que ambas as constantes de sensibilidade A e as condições em que elas são válidas (por exemplo, as condições em que uma reação se processa quantitativamente) são algumas vezes determinadas teórica ou experimentalmente (N. A.)

c Spiked (N.T.).

${ }^{\mathrm{d}}$ Métodos gráficos ainda são às vezes aplicados. Ultimamente tem-se usado redes neurais para ajustar modelos de calibração, particularmente para relações não lineares (N.A.).

${ }^{\mathrm{e}}$ Standard-free (N.T.).

${ }_{\mathrm{f}}^{\mathrm{f}}$ No documento original da IUPAC usa-se "sum of squares of deviations". Preferimos a tradução soma quadrática residual, porque se trata de desvios dos valores observados em relação aos valores dados pelo modelo (N.T.).

${ }^{\mathrm{g}}$ A soma quadrática vem da função de verossimilhança, $\mathrm{L}=(2 \pi)^{-1} \sigma_{1}^{-1} \sigma_{2}^{-1} \ldots \sigma_{\mathrm{n}}^{-1} \exp \left(-1 / 2 \mathrm{SQ} \mathrm{Q}_{\mathrm{r}}\right)$, que é o produto das probabilidades de que os valores medidos $y_{i}$ correspondam às estimativas $\hat{y}_{i}$. Quando $\mathrm{L}$ é máximo, $S Q_{r}$ torna-se mínimo (N.A.).
} 
(1) Os erros estão somente (ou principalmente) nos valores medidos para a variável dependente, $y$ :

$A \sigma_{x} \ll \sigma_{y}$

Além disto, os erros $\sigma_{y}$ são constantes nos diversos pontos da calibração (Homoscedasticidade):

$\sigma_{y 1}^{2}=\sigma_{y 2}^{2}=\ldots \sigma_{y n}^{2}=\sigma_{y}^{2}$

ou, se expressados pelas estimativas de $\sigma$,

$s_{y_{1}}^{2} \stackrel{\alpha}{=} s_{y_{2}}^{2} \stackrel{\alpha}{=} \stackrel{\alpha}{=} s_{y_{n}}^{2}=s_{y}^{2}$

onde $\stackrel{\alpha}{=}$ significa igualdade para um dado risco estatístico de erro, $\alpha$. Somente se houver homoscedasticidade, e se os erros em $x$ puderem ser desprezados de acordo com a Eq. 13, é que o critério dos mínimos quadrados se reduz a:

$\sum_{i=1}^{m} d{ }_{y i}^{2} \stackrel{!}{=} \min$

e o critério clássico dos mínimos quadrados gaussianos, normais ou ordinários (MQ, MQN ou MQO) pode ser aplicado.

(2) Quando os erros $\sigma_{y}$ nas medidas variam (isto é, as Eqs. 14 não são válidas), a heteroscedasticidade tem de ser admitida e o critério de mínimos quadrados da Eq. (12) se transforma em:

$$
\sum_{i=1}^{m}\left(d_{y i} / \sigma_{y i}\right)^{2} \stackrel{!}{=} \min
$$

Deste critério resulta o modelo de mínimos quadrados ponderados (MQP), como se mostrará mais adiante.

(3) No caso mais geral, se ambas as variáveis estão sujeitas a erros, e portanto a Eq.(13) não é obedecida, tem-se

$\sigma_{i}^{2}=\sigma_{y i}^{2}+A^{2} \sigma_{x i}^{2}$

Neste caso, em que há erro no valor medido e na quantidade ou concentração do analito, deve-se minimizar a soma dos termos $d_{x+y}^{2}$ (Figura 3), e fazer o ajuste por mínimos quadrados ortogonais. Os diferentes modelos de mínimos quadrados que podem ser calculados são mostrados esquematicamente na Figura 3.

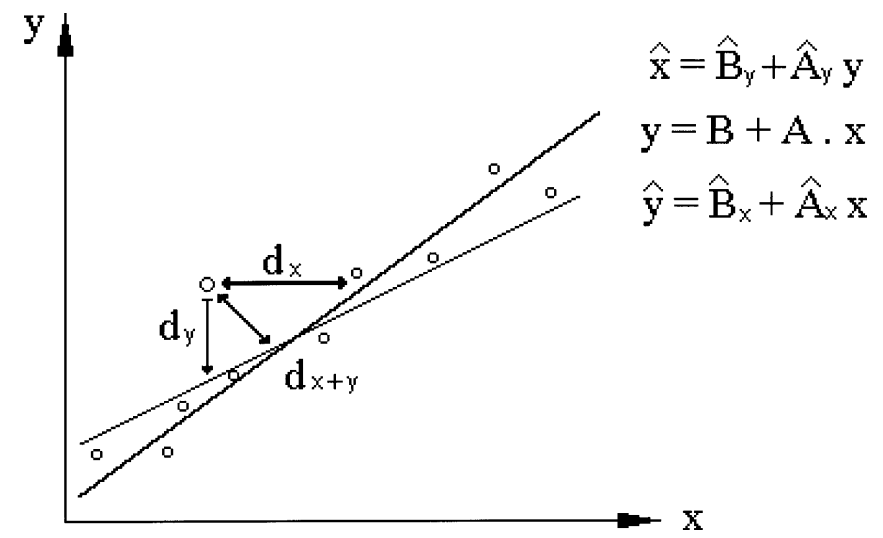

Figura 3. Diferentes ajustes por mínimos quadrados. $\hat{B}_{y}, \hat{B}_{x}, \hat{A}_{y}$ e $\hat{A}_{x}$ são as estimativas de $B_{y}, B_{x}, A_{y}$ e $A_{x}$

A escolha do modelo a ser usado na calibração analítica depende da concordância com as condições mencionadas acima e do procedimento de calibração.
De acordo com a Eq. (2), as calibrações experimentais são realizadas principalmente fazendo-se determinações em uma série de amostras de calibração contendo o analito de interesse em quantidades adequadas. Se possível, os analistas usam materiais cujas concentrações são conhecidas com o máximo de confiança, isto é, com alta precisão e exatidão. Na prática analítica, usam-se como amostras de calibração materiais de referência certificados, padrões contendo um ou vários componentes, e materiais padrão sintéticos.

As quantidades ou concentrações das amostras de calibração são tomadas como "verdadeiras" e isentas de erro, ou então admite-se que, de acordo com a Eq. (13), os erros aleatórios de $x$ são desprezíveis em relação aos de $y$. Nessas condições, deve-se usar a função de calibração:

$y=B_{x}+A_{x} x+e_{y}$

Se houver homoscedasticidade, os parâmetros $B_{x} e A_{x}$ são estimados pelo algoritmo gaussiano (normal) dos mínimos quadrados ${ }^{14,15}$ :

$\hat{A}_{x}=Q_{x y} / Q_{x x}$

$\hat{B}_{x}=\left(\sum y-\hat{A}_{x} \sum x\right) / m$

onde $m$ é o número total de experimentos da calibração (índice $j$ ) usados para construir a função de calibração, com os seguintes somatórios:

$Q_{x x}=\sum\left(x_{j}-\bar{x}\right)^{2}=\sum x_{j}^{2}-\left(\sum x_{j}\right)^{2} / m$

$Q_{y y}=\sum\left(y_{j}-\bar{y}\right)^{2}=\sum y_{j}^{2}-\left(\sum y_{j}\right)^{2} / m$

$Q_{x y}=\sum\left(x_{j}-\bar{x}\right)\left(y_{j}-\bar{y}\right)=\sum\left(x_{j} y_{j}\right)-(m \bar{x} \bar{y})$

O coeficiente de correlação,

$r_{x y}=Q_{x y} / \sqrt{Q_{x x} Q_{x y}}$

que é uma medida da relação entre duas variáveis aleatórias, não tem nenhum significado na calibração feita nas condições mencionadas, porque os valores de $x$ em um experimento de calibração não são grandezas aleatórias.

Nota 2: O coeficiente de correlação $r_{x y}$ faz sentido para a relação entre variáveis aleatórias, mas não deve ser usado na calibração.

Para avaliação das medidas analíticas, normalmente se aplica a função inversa da função de calibração [Eq. (18)], dada por:

$\hat{x}=\left(y-\hat{B}_{x}\right) / \hat{A}_{x}$

desde que as condições (1) e (2) mencionadas acima sejam satisfeitas.

Nota 3: Na realidade, a relação entre os valores medidos, $y$, e a quantidade ou concentração do analito, $x$, deve ser caracterizada por um modelo de calibração tridimensional ${ }^{2}$ :

$y=f\left(x_{\text {verdad }}, x_{\text {estim }}\right)$

onde $x_{\text {verdad }}$ é a concentração do material de referência (certificado) usado na calibração, que é considerada como "verdadeira" ("sem erro"). Já a variável $x_{\text {estim }}=\hat{x}$ é uma variável aleatória, afetada por erros. Não se pode determinar, $a$ priori, se a condição da Eq.(13) é satisfeita ou não por $x_{\text {estim }}$ 
Do modelo tridimensional (i) resultam as três relações bidimensionais seguintes:

a função de calibração:

$y=f_{C}\left(x_{\text {verdad }}\right)+e_{y}$

(por exemplo, de acordo com a Eq. 18);

a função de avaliação analítica,

$x_{\text {estim }}=f_{A}(y)+e_{x}$

(por exemplo, de acordo com a Eq. 23); e

a função de validação (função de tendência ${ }^{h}$, função de recuperação):

$x_{\text {estim }}=f_{V}\left(x_{\text {verdad }}\right)+e_{x}$

que caracteriza a exatidão dos resultados analíticos. Somente para as funções de validação em que $x_{\text {estim }}=x_{\text {verdad }}$, a relação tridimensional (i) torna-se bidimensional e a calibração comum, por mínimos quadrados, se justifica.

\section{Erros na calibração linear e sua estimativa por mínimos quadra-} dos ordinários

Fundamentalmente, as incertezas dos valores de $y$ estimados pela calibração, de acordo com a Eq.(18), por exemplo, e dos resultados analíticos (quantidades ou concentrações dos analitos) estimados através dos modelos de calibração, por exemplo de acordo com a Eq.(23), diferem entre si. A incerteza nos valores de $y$ na calibração é caracterizada pelo intervalo de confiança, $\operatorname{cnf}(y)=\Delta y$, enquanto a incerteza nos valores estimados de $x$ é caracterizada pelo intervalo $\underline{\text { de previsão }}, \operatorname{prv}(x)=\Delta y_{p}$. O $\underline{\text { intervalo de previsão }}$ de um valor medido de $y, \operatorname{prv}(y)=\Delta y$, também tem o seu papel na definição do valor crítico (limite de decisão), do limite de detecção, e do limite de quantificação ${ }^{1,16-19}$.

A precisão na calibração é caracterizada pelos seguintes erros especiais:

Desvio padrão residual

$s_{y, x}=\sqrt{\frac{\sum\left(y_{j}-\hat{y}_{j}\right)^{2}}{m-2}}=\sqrt{\frac{\sum\left(y_{j}-\hat{B}_{x}-\hat{A}_{x} x_{j}\right)^{2}}{m-2}}$.

Note que o número de graus de liberdade é $g l=m-2$, neste modelo com dois parâmetros, dado pela Eq.(8). Para uma calibração linear passando pela origem, dada pela Eq.(9), $g l=m-1$.

Estimativa do desvio padrão para a estimativa da interseção (branco) $B$

$s_{B}=s_{y, x} \sqrt{\frac{1}{m}+\frac{\bar{x}^{2}}{Q_{x x}}}$

Estimativa do desvio padrão da estimativa do coeficiente angular A

$s_{A}=s_{y, x} / \sqrt{Q_{x x}}$
Estimativa do desvio padrão da estimativa de uma média $\hat{y}_{e}$ na posição $x_{i}$

$s_{\hat{y}_{e}}=s_{y, x} \sqrt{\frac{1}{m}+\frac{\left(x_{i}-\bar{x}\right)^{2}}{Q_{x x}}}$

Estimativa do desvio padrão de um valor previsto $\hat{y}_{p}$ na posição $x_{i}$

$s_{\hat{y}_{p}}=s_{y, x} \sqrt{1+\frac{1}{m}+\frac{\left(x_{i}-\bar{x}\right)^{2}}{Q_{x x}}}$

Estimativa do desvio padrão de uma média $\hat{\bar{y}}_{p}$ prevista a partir de $n$ repetições na posição $x_{i}$

$s_{\hat{\bar{y}}_{p}}=s_{y, x} \sqrt{\frac{1}{n}+\frac{1}{m}+\frac{\left(x_{i}-\bar{x}\right)^{2}}{Q_{x x}}}$

Estimativa do desvio padrão de uma média $\hat{\bar{x}}_{p}$ prevista a partir de $n$ repetições na posição $x_{i}$

$s_{\hat{\bar{x}}_{p}}=s_{y, x} / A \sqrt{\frac{1}{n}+\frac{1}{m}+\frac{\left(y_{i}-\bar{y}\right)^{2}}{A^{2} Q_{x x}}}$

A região de confiança, $R C$, da reta de calibração, mostrada na Figura 4, é dada por

$R C=\hat{y} \pm s_{\hat{y}_{e}} \sqrt{2 F_{\alpha ; g l 1=2 ; g l 2=m-2}}$

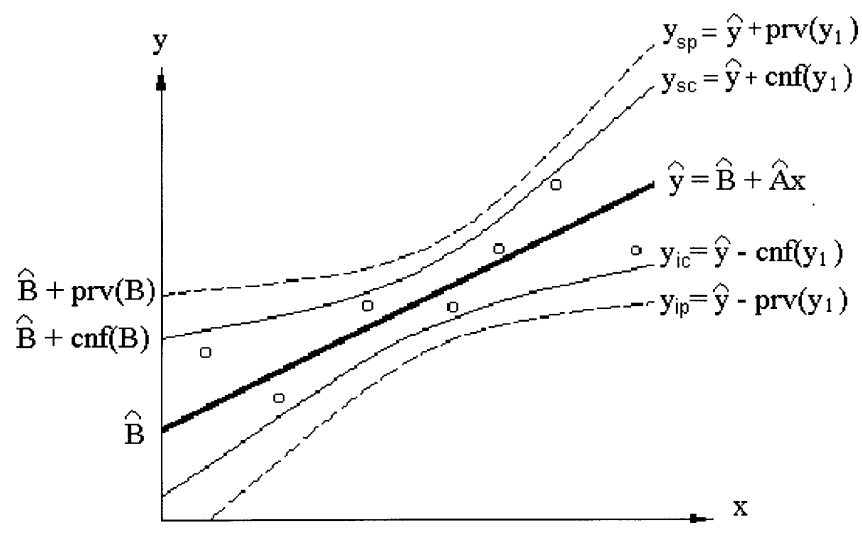

Figura 4. Reta de calibração com as bandas relevantes de confiança e de previsão: $y_{s p}$ é o limite superior de previsão, $y_{s c}$ é o limite superior de confiança, $y_{i p}$ é o limite inferior de previsão e $y_{i c}$ é o limite superior de confiança

Os seguintes intervalos, que resultam das Eqs. (24) a (30), são de interesse prático:

Intervalo de confiança da interseção (branco) $B$

$\operatorname{cnf}(B)=B \pm s_{B} t_{\alpha, g l=m-2}$

Intervalos de previsão prv(B) de um único valor de B (Eq.(33)) e de uma média $\bar{B}$ obtida a partir de $n$ repetições (Eq.(34)). Este último é importante para a estimativa do limite de detecção a partir de brancos. 
$\operatorname{prv}(B)=B \pm s_{y, x} t_{\alpha, g l=m-2} \sqrt{1+\frac{1}{m}+\frac{\bar{x}^{2}}{Q_{x x}}}$

$\operatorname{prv}(\bar{B})=\bar{B} \pm s_{y, x} t_{\alpha, g l=m-2} \sqrt{\frac{1}{n}+\frac{1}{m}+\frac{\bar{x}^{2}}{Q_{x x}}}$

Intervalo de confiança para a estimativa de uma média $\hat{\bar{y}}_{i c}$ na posição $x_{i}$

$\operatorname{cnf}\left(\bar{y}_{i}\right)=\hat{\bar{y}}_{i e} \pm s_{y, x} t_{\alpha, g l=m-2} \sqrt{\frac{1}{m}+\frac{\left(x_{i}-\bar{x}\right)^{2}}{Q_{x x}}}$

Intervalo de previsão de um valor previsto $\hat{y}_{i p}$, na posição $x_{i}$

$\operatorname{prv}\left(y_{i}\right)=\hat{y}_{i p} \pm s_{y, x} t_{\alpha, g l=m-2} \sqrt{1+\frac{1}{m}+\frac{\left(x_{i}-\bar{x}\right)^{2}}{Q_{x x}}}$

Intervalo de previsão de uma média $\hat{\bar{y}}_{i p}$ obtida a partir de $n$ repetições na posição $x_{i}$

$\operatorname{prv}\left(\bar{y}_{i}\right)=\hat{\bar{y}}_{i p} \pm s_{y, x} t_{\alpha, g l=m-2} \sqrt{\frac{1}{n}+\frac{1}{m}+\frac{\left(x_{i}-\bar{x}\right)^{2}}{Q_{x x}}}$

Intervalo de previsão de uma média $\hat{\bar{x}}_{i p}$ obtida a partir de $n$ repetições, para um valor medido $y_{i}$

$\operatorname{prv}\left(\bar{x}_{i}\right)=\hat{\bar{x}}_{i p} \pm\left(\frac{s_{y, x}}{A}\right) t_{\alpha, g l=m-2} \sqrt{\frac{1}{n}+\frac{1}{m}+\frac{\left(y_{i}-\bar{y}\right)^{2}}{A^{2} Q_{x x}}}$

\section{Estimativa por mínimos quadrados ponderados (MQP)}

Nos casos em que a homoscedasticidade descrita pela Eq.(14a) não se verifica, a estimativa do desvio padrão $s_{y}$ muitas vezes é uma função do valor medido: $s_{y}=f(y)$ ou, estritamente falando, $\sigma y=$ $f[E(y)]$, o que significa que o desvio padrão pode ser uma função do valor esperado de $y$. Nesse caso, o sistema de calibração é heteroscedástico e deve-se aplicar o ajuste por mínimos quadrados ponderados ${ }^{20,21}$. As diferentes variâncias nos pontos da calibração são levadas em conta usando-se ponderação. Na ponderação pela variância, os pesos são:

$w_{y i}=\frac{1 / s_{y i}^{2}}{\left(\sum_{i=1}^{p} 1 / s_{y i}^{2}\right) / p}$

para $p$ pontos de calibração (índice $i$ ), e o critério de minimização da Eq. (16) torna-se:

$\sum w_{y i} d_{y i}^{2} \stackrel{!}{=} \min$

Os coeficientes da calibração são calculados por analogia com as Eqs (19) e (20), introduzindo-se os pesos $w_{i}=w_{y i}$ :

$$
\begin{aligned}
A_{x, w} & =\frac{m \sum w_{i} x_{i} y_{i}-\sum w_{i} x_{i} \sum w_{i} y_{i}}{m \sum w_{i} x_{i}^{2}-\left(\sum w_{i} x_{i}\right)^{2}} \\
B_{x, w} & =\left(\sum w_{i} y_{i}-A_{x, w} \sum w_{i} x_{i}\right) / m
\end{aligned}
$$

A estimativa do desvio padrão residual é $s_{y, x, w}=\sqrt{\frac{\sum w_{i}(y-\hat{y})^{2}}{m-2}}$

Outras medidas de incerteza podem ser estimadas por analogia com as Eqs (25) a (38). Alguns programas computacionais de análise de regressão permitem que o usuário forneça uma estimativa da função $\sigma y=f[E(y)]$, que é então usada para a ponderação.

A decisão de usar (ou não) mínimos quadrados ponderados pode ser tomada a partir de testes estatísticos ou de modelos teóricos.

\section{Ajuste por mínimos quadrados quando há erros em ambas as variáveis (MQ ortogonais)}

Três retas de calibração são dadas na Figura 3. Uma é o modelo para estimar $y$ a partir de valores de $x$ (praticamente) isentos de erro. Esta relação é comumente usada para calibração por mínimos quadrados ordinários:

$\hat{y}=\hat{B}_{x}+\hat{A}_{x} x$

Outra reta é um modelo formulado para estimar $x$ a partir de valores de $y$, sob a condição de que $s_{y}<A s_{x}$ :

$\hat{x}=\hat{B}_{y}+\hat{A}_{y} y$

Nota 4: Deve-se notar explicitamente que a Eq.(45) não é a função inversa da Eq. (44) e portanto $\hat{B}_{y} \neq-\hat{B}_{x} / \hat{A}_{x}$ e $\hat{A}_{y} \neq 1 / \hat{A}_{x}$

Nota 5: Em geral, a Eq.(45) não tem relevância prática em Química Analítica. Ela serve apenas como uma forma de estimar a linha de calibração ortogonal dada pela Eq. (46).

A função de calibração quando existem erros em ambas as variáveis,

$\hat{y}=\hat{B}+\hat{A} x$

deve ser determinada por mínimos quadrados ortogonais, onde os erros na variável independente e na variável dependente são minimizados simultaneamente. Esta calibração deve ser aplicada quando tanto os valores medidos $y$ quanto os valores analíticos $x$ (concentrações) forem afetados por erros. $\mathrm{O}$ modelo dado pela Eq. (46) não pode ser determinado diretamente. É preciso determiná-lo por aproximações. Por exemplo, o coeficiente angular $A$ pode ser estimado pela média geométrica (MG) das retas dadas pelas Eq.(44) e (45) ${ }^{13}$, usando-se:

$\hat{A}=\tan \left[1 / 2\left(\tan ^{-1} \hat{A}_{x}+\tan ^{-1} \hat{A}_{y}\right)\right]$

onde $\hat{A}_{x}$ é dado pela Eq.(19) e $\hat{A}_{y}=Q_{x y} / Q_{y y}$. A estimativa de $B$ é obtida a partir da Eq. (46) por analogia com a Eq. (20). Outro procedimento para estimar $A$ foi proposto por Wald ${ }^{22}$ :

$A=\frac{\sum_{i=1}^{g} y_{i}-\sum_{j=h}^{m} y_{j}}{\sum_{i=1}^{g} x_{i}-\sum_{j=h}^{m} x_{j}}$

onde $m$ é o número de medidas de calibração, $g=m / 2=h-1$ quando $m$ é par, e $g=(m+1) / 2=h$ quando $m$ é ímpar. Deve-se mencionar também que a primeira componente principal, $p_{1}$, obtida em uma análise de componentes principais (ACP $)^{23-25}$ é uma boa aproximação da linha de calibração ortogonal. 
A ref. 28 apresenta uma comparação de vários modelos aproximados para a calibração ortogonal. Outros estudos também tratam do mesmo problema ${ }^{29-31}$.

\section{TESTES ESTATÍSTICOS}

Para se aplicar o modelo de calibração adequado, é importante testar se as condições dadas na seção Modelo linear de calibração são satisfeitas. Inicialmente, pode-se usar um programa computacional para examinar visualmente os erros residuais de um dado modelo de calibração, obtendo-se assim uma informação preliminar sobre as características destes erros. Gráficos típicos, como os mostrados na Figura 5, também indicam quais os testes que devem ser realizados, tais como os de aleatoriedade, de normalidade, de linearidade, de homoscedasticidade, etc.

\section{Linearidade}

Pode-se avaliar se o modelo linear é adequado examinando-se a distribuição dos resíduos ao longo dos valores de $x$. Na Figura 5a os erros distribuem-se aleatoriamente em torno da linha zero, indicando que o modelo linear é adequado. Na Figura 5b pode-se notar que os erros apresentam desvios sistemáticos e, mesmo no caso dado onde os desvios alternam de maneira real, isto é uma indicação de que o modelo linear é inadequado e que deve-se escolher um modelo não-linear. A hipótese de linearidade pode ser testada de duas maneiras.

(a) a priori (sem levar em consideração nenhum modelo não-linear), comparando-se o desvio padrão residual, $s_{y, x}$, obtido a partir da Eq. 24 com o desvio padrão dos valores $y_{i j}$ em relação a suas médias $\bar{y}_{i}$, que é dado por $\mathrm{s}_{y}$ :

$\hat{F}=\frac{s_{y, x}^{2}}{s_{y}^{2}}=\frac{\sum_{i=1}^{p} m_{i}\left(\bar{y}_{i}-\hat{y}_{i}\right)^{2} /(p-2)}{\sum_{i=1}^{p} \sum_{j=h}^{m i}\left(y_{i j}-\bar{y}_{i}\right)^{2} /(m-p)}$

( $m_{i}$ é o número de medidas repetidas nos $p$ pontos de calibração;

$\sum_{i=1}^{p} m_{i}=m ;$ normalmente $m_{1}=m_{2}=\ldots=m_{p}$, e $\left.p \cdot m_{i}=m\right)$. O teste é feito comparando-se o quociente da Eq. (49) com $\mathrm{F}_{\alpha, g l l=p-2, g l 2=m-p}$.

(b) a posteriori (em relação a um dado modelo não-linear), comparando-se o desvio padrão residual do modelo linear com o desvio padrão residual do modelo não linear:

$\hat{F}=\frac{s_{y, x, \operatorname{lin}}^{2}}{s_{y, x, n \operatorname{lin}}^{2}}=\frac{\sum_{i=1}^{m}\left(y_{i}-\hat{y}_{i}\right)^{2} /(m-2)}{\sum_{i=1}^{m}\left(y_{i}-\hat{y}_{i}\right)^{2} / g l_{n l i n}}$

O número de graus de liberdade para os modelos lineares é $g l_{\text {lin }}$ $=m-2$ se forem estimados dois parâmetros (Eq. (8)), ou $m-1$ se for estimado apenas um (Eq. (9)). No caso não-linear, o número de graus de liberdade depende do modelo escolhido. Por exemplo, para uma equação quadrática $y=a+b x+c x^{2}, g l_{n l i n}=m-3$. De acordo com Mandel ${ }^{32}$, pode-se fazer um teste apropriado para a Eq. (50) comparando-se

$\hat{F}=\frac{s_{y, x, l i n}^{2}-s_{y, x, n l i n}^{2}}{s_{y, x, n l i n}^{2}}$

$\operatorname{com} F_{\alpha, g l l=1, g l 2 \text {-fnlin. }}$. Se $\hat{F} \geq F_{\alpha ; g l ; g l l}$, o modelo linear não pode ser aplicado.

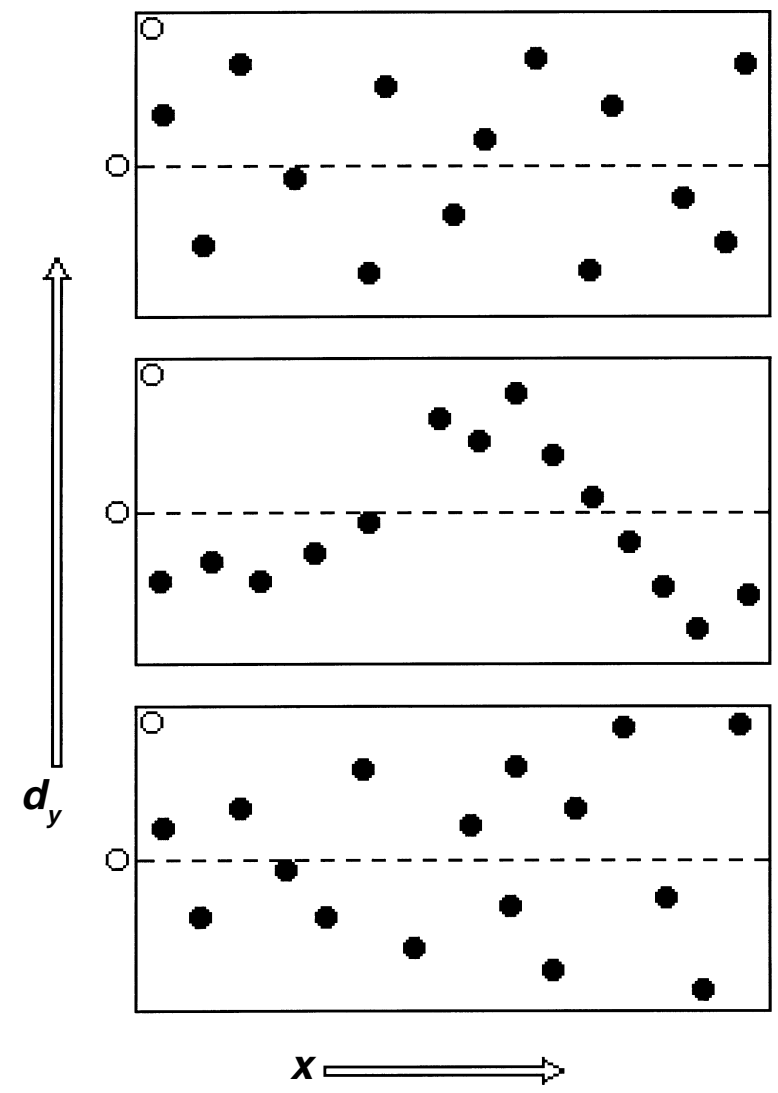

a

Figura 5. Gráficos típicos dos desvios residuais

\section{Homoscedasticidade}

Pode-se verificar se a variância não é constante através de gráficos de resíduos como o da Figura 5c. Nestes gráficos, $d_{y}$ freqüentemente é uma função de $x$ com aparência afunilada, como aparece na figura $5 c$. Nesses casos, a homoscedasticidade pode ser avaliada de maneira simples com o teste de Hartley $^{33}$ (para valores de mi iguais nos $p$ pontos de calibração):

$\hat{F}=s_{\max }^{2} / s_{\min }^{2}$

Em situações mais obscuras que a representada na Figura 5c, é preciso aplicar o teste de Bartlett para homogeneidade de variâncias ${ }^{34}$ :

$\hat{\chi}^{2}=2,303 / c\left(g l \log s^{2}-\sum_{i=1}^{p} g l_{i} \log s_{i}^{2}\right)$

onde $g l=m-p=\sum g l_{i}$ é o número total de graus de liberdade (mais uma vez, $p$ é o número de pontos de calibração, em cada um dos quais são realizadas $m_{i}$ medidas repetidas $), s^{2}=\sum\left(g l_{i} s_{i}^{2} / g l\right)$ é a variância ponderada, $s_{i}^{2}$ a variância do $i$-ésimo grupo de pontos, com $g l_{i}$ graus de liberdade, e $c$ é uma constante de correção calculada a partir de $c=\left\{\sum\left(1 / g l_{i}-1 / g l\right)[3(m-1)]+1\right\}$ quando o número de graus de liberdade é pequeno.

$\hat{\chi}^{2}$ deve ser comparado com o valor crítico da distribuição $\chi_{\alpha ; g l}^{2}$ e se $\hat{\chi}^{2} \geq \chi_{\alpha ; g l}^{2}$, a hipótese nula $s_{1}=s_{2}=\ldots=s_{p}$ deve ser rejeitada.

\section{Teste dos parâmetros de calibração}

Em alguns casos, pode ser útil comparar os valores dos parâmetros $A$ e $B$ obtidos experimentalmente com valores $\alpha$ e $\beta$ teoricamente esperados. A comparação é realizada com um teste $t$ de Student:

$\hat{t}=|A-\alpha| / s_{A}$ 
$\hat{t}=|B-\beta| / s_{B}$

Em relação à validação, as hipóteses nulas $\alpha=1$ e $\beta=0$ são as de maior interesse. Se $\hat{t} \geq t_{\alpha ; g l}$, a hipótese nula correspondente deve ser rejeitada.

\section{VALIDAÇÃO DA CALIBRAÇÃo}

Como regra geral, a exatidão dos resultados analíticos é garantida por experimentos de validação. A validação de um procedimento de calibração é baseada na função de validação (função de recuperação) $x_{\text {estim }}=f\left(x_{\text {verdad }}\right)$ (veja Eq.(iv), da nota 3 ). Para se avaliar a exatidão dos resultados analíticos, duas maneiras práticas são usadas:

Análise de material de referência certificado (MRC) com teores "verdadeiros". A função de validação é estimada por regressão MQ normal:

$$
E\left(x_{e s t i m}\right)=b+a x_{v e r d a d}
$$

onde $a$ e $b$ são os coeficientes de validação, significando, analiticamente, uma tendência ${ }^{i}$ constante $(b)$ e uma tendência proporcional (a). As estimativas de $a$ e de $b$ podem ser obtidas das Eqs (19) e (20). A ausência de tendências nos resultados analíticos pode ser verificada usando-se as Eqs (54) e (55) para testar as hipóteses nulas $a=1 \mathrm{e}$ $b=0$.

Desvios sistemáticos também são detectados se os intervalos de confiança dos coeficientes de validação não contiverem os valores 0 (para $b$ ) ou 1 (para $a$ ). Isto é:

(i) uma tendência aditiva se $b+\Delta b<0$ ou $b-\Delta b>0 \quad(b>|\Delta b|)$

(ii) uma tendência proporcional se $a+\Delta a<1$ ou $a-\Delta a>1$

$$
(a>|\Delta a|)
$$

Análise por dois métodos independentes de uma determinada série de amostras de teste com concentrações que variam gradualmente. A exatidão deve ser verificada para o método I, enquanto o método II é tido como exato. A função de recuperação apropriada é:

$x_{I, \text { estim }}=b+a x_{I I, \text { verdad }}$

Como neste procedimento tanto $x_{I, \text { estim }}$ quanto $x_{I I, v e r d a d}$ estão sujeitos a erros, deve-se usar um ajuste por mínimos quadrados ortogonais (Eqs (47) e (48)), uma análise de componentes principais ou um ajuste robusto. Os testes de desvios significativos das hipóteses $a=1$ e $b=0$ são feitos como indicado acima.

\section{CALIBRAÇÃO ROBUSTA}

Se as condições básicas para o uso do ajuste por mínimos quadrados não forem satisfeitas, ou se surgirem pontos de calibração com desvios muito grandes ("pontos anômalos" ou, de forma mais exata, pontos influentes ${ }^{i}$ ), o método dos mínimos quadrados ordinários falha, isto é, os parâmetros estimados são tendenciosos e portanto não representativos da relação entre $x$ e $y$. A normalidade dos valores medidos freqüentemente pode ser obtida por meio de uma transformação adequada, mas quando existirem pontos de calibração anômalos ${ }^{35}$ deve-se fazer uma calibração robusta. No caso mais simples, estimativas robustas dos parâmetros podem ser baseadas em medianas. Todos os coeficientes angulares entre dois pontos de calibração quaisquer são calculados através de $A_{i j}=\frac{\left(y_{j}-y_{i}\right)}{\left(x_{j}-x_{i}\right)}$, para $j>i$. Os valores $A_{i j}$ são colocados em ordem crescente, e sua mediana é tomada como estimativa do coeficiente angular médio:

$\bar{A}=\operatorname{med}\left\{A_{i j}\right\}$

A estimativa da interseção é dada por

$\bar{B}=\operatorname{med}\left\{y_{i}-\bar{A} x_{i}\right\}$

A forma de se calcular as estimativas das variâncias e dos intervalos de confiança na calibração robusta pode ser encontrada na literatura $^{35,36}$.

Nota 6: Na maioria dos casos, a calibração robusta se torna necessária por causa da presença de pontos de calibração anômalos (pontos influentes). É aconselhável, por isso, verificar se a relação pode ser considerada linear e se os resíduos podem ser considerados aleatórios.

A relação entre os modelos de calibração mais importantes e as condições em que eles devem ser aplicados está representada no esquema da Figura 6. Como já foi ressaltado acima, o ajuste por mínimos quadrados ordinários só deve ser aplicado quando os valores medidos forem independentes, distribuídos normalmente, isentos de anomalias ou pontos influentes, e caracterizados por erros homoscedásticos. Além disto, os valores da grandeza analítica (concentração) devem ser praticamente isentos de erros.

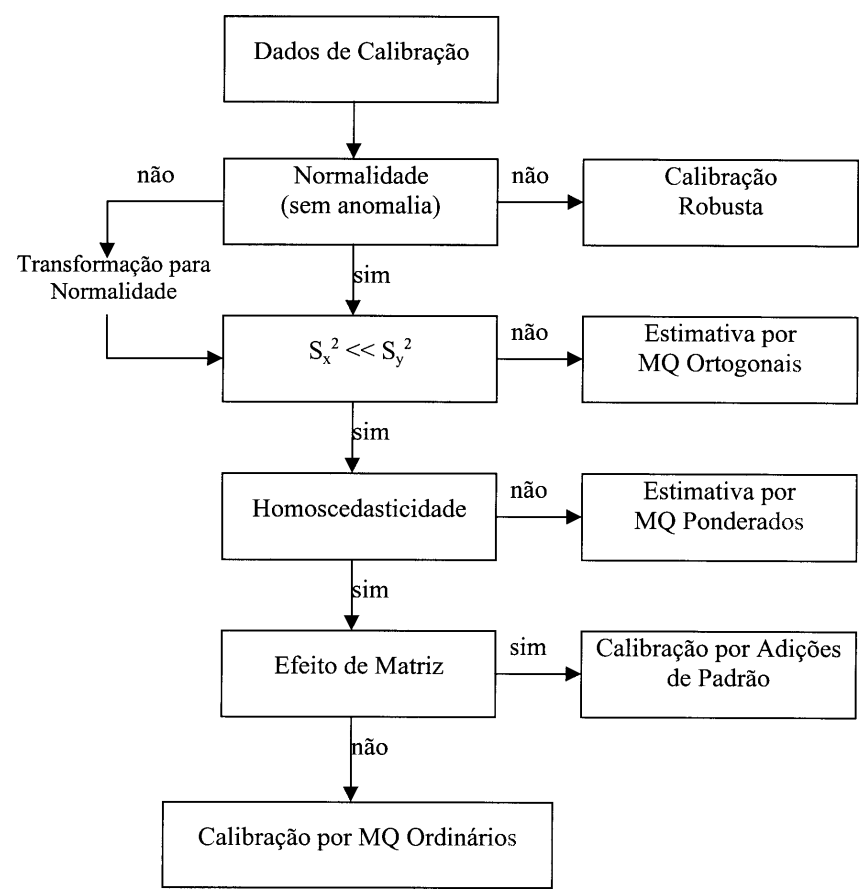

Figura 6. Relação entre diferentes modelos de calibração e as condições estatísticas e químicas que os dados devem satisfazer

${ }^{\mathrm{i}} \operatorname{bias}$ (N.T.)

${ }^{j}$ No original, outliers e leverage points, respectivamente. Alguns autores preferem traduzir outliers como valores discrepantes ou valores suspeitos (N.T.). 
Do ponto de vista químico, em casos onde aparecerem efeitos de matriz e não se dispuser de um material de referência certificado apropriado, a calibração pode ser realizada na própria matriz da amostra por adições de padrão.

\section{CALIBRAÇÃO POR ADIÇÕES DE PADRÃO}

Quando existirem (ou forem esperados) efeitos de matriz e não se dispuser de amostras de calibração com uma matriz semelhante ${ }^{k}$, o método de adições de padrão (MAP) pode ser o mais indicado. MAP é utilizado com frequiência em sistemas ambientais e bioquímicos e na análise de ultratraços em geral. Adicionando-se soluções-padrão à amostra, produz-se na série de calibração um comportamento semelhante ao da amostra, desde que o analito no padrão adicionado esteja na mesma forma da amostra.

O modelo de adições de padrão é baseado no pré-requisito de que o branco não apresenta valor estatisticamente diferente de zero ou que esse valor pode ser eliminado, Eq.(9). Se $x_{0}$ é a concentração inicial do analito na amostra que está sendo investigada, então:

$y_{0}=A_{x} x_{0}$

onde $y_{0}$ é o valor medido para a amostra sem adição de padrão. Quantidades conhecidas, $x$, do analito são adicionadas à amostra, de preferência em teores equimolares de $x_{i}$, na faixa de $x_{1} \approx x_{0}, x_{2}=2 x_{1}, \ldots$, $x_{p}=p x_{1}$ (normalmente com $p=3$ ou 4). Para isto, alguma idéia da concentração inicial $x_{0}$ é necessária.

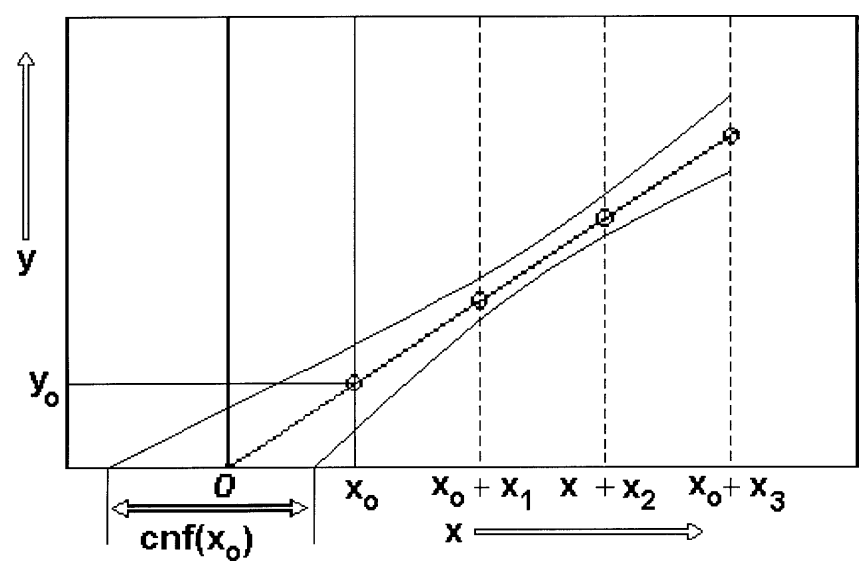

Figura 7. Calibração por adições de padrão

A função de calibração por adição de padrão (AP) é estimada por mínimos quadrados. O coeficiente angular é dado por:

$A_{x}=\frac{\left(\bar{y}_{p}-\bar{y}_{0}\right)}{x_{p}}$

Este procedimento só se justifica se a sensibilidade da determinação das espécies na amostra for igual à das espécies adicionadas:

$A_{x}=\frac{\Delta y}{\Delta x}=\frac{y_{0}}{x_{0}}=\frac{\left(\bar{y}_{p}-\bar{y}_{0}\right)}{x_{p}}$, onde $p$ é o número de amostras com adições de padrão. A solução para $x$ é dada pela função analítica AP, cuja extrapolação para $y=0$ fornece:
$x_{0}=\frac{\bar{y}_{0}}{A_{x}}=\frac{x_{p} \cdot \bar{y}_{0}}{\bar{y}_{p}-\bar{y}_{0}}$

com o intervalo de confiança:

$\operatorname{cnf}\left(x_{0}\right)=x_{0} \pm s_{y, x} t_{\alpha ; g l=m-2} / A_{x} \sqrt{\frac{1}{m}+\frac{\left(-x_{0}-x_{p} / 2\right)^{2}}{Q_{x x}}}$

que é mais largo que o intervalo da calibração normal (Eq.35, dividida por $A_{x}$ ), por causa da extrapolação até $x_{i}=-x_{0}$. O número de medidas de calibração, $m$, é dado por $m_{0}+p m_{i}$ ou $m_{0}+\Sigma^{p} m_{i}$, sendo $m_{0}$ o número de medições de $y_{0}$.

Quando o branco apresentar um valor diferente de zero, deve-se estimá-lo a partir de um número suficientemente alto de medidas de branco, e então corrigir os valores medidos em relação a ele. Para garantir um modelo de calibração adequado por adições de padrão, deve-se realizar $p \geq 2$ adições de padrão. Uma única adição (repetida $m_{i}$ vezes) só pode ser feita quando se tiver certeza de que o modelo linear é adequado. Em geral, a linearidade pode ser testada com as Eqs (49) - (51).

Se a linearidade na faixa $x<x_{0}$ tiver sido apenas admitida, e não verificada experimentalmente, a calibração por adições de padrão torna-se um método pouco confiável. No entanto, praticamente não existe alternativa, quando se suspeita da presença de efeitos de matriz.

\section{SUMÁRIO}

Os fundamentos da calibração em Química Analítica são apresentados. Os dados de calibração podem apresentar diferentes características estatísticas, de acordo com o método analítico. Dependendo da normalidade dos dados, da variável independente (a concentração dos padrões de calibração) ser isenta de erro e da condição de homoscedasticidade, a calibração pode ser realizada pelo método dos mínimos quadrados simples, normais ou ordinários (MQ, MQN ou MQO), pelos mínimos quadrados ponderados (MQP), pelos mínimos quadrados ortogonais (tratamento dos erros em ambas as variáveis) ou por calibração robusta, como mostra o fluxograma da Figura 6.

Na maioria dos casos práticos, pode-se usar a calibração por mínimos quadrados ordinários. Quando dois métodos são comparados para fins de validação, os erros em ambas as variáveis devem ser considerados e, portanto, deve-se aplicar o ajuste por mínimos quadrados ortogonais.

\section{AGRADECIMENTOS}

Os tradutores agradecem a E. A. G. Zagatto por críticas e sugestões e ao CNPq pela bolsa de Produtividade em Pesquisa.

\section{REFERÊNCIAS}

1. Currie, L.A.; IUPAC Commission on Analytical Nomenclature; Pure Appl. Chem. 1995, 67, 1699.

2. Danzer, K.; Fresenius' J. Anal. Chem. 1995, 351, 30

3. Moseley, H.; Phil. Mag. 1913, 26, 1024.

4. Kovats, E.; Helv. Chim. Acta 1958, 41, 1915; Anal. Chem. 1964, 36, 31A.

5. Colthup, N.B.; Daly, L.H.; Wiberly, S.E.; Introduction to Infrared and Raman Spectroscopy, Academic Press: New York, London, 1975.

6. De Gregorio, P.; Savastano, G.; Spektrum des Eisens von 2206 bis $4656 \AA$ mit Analysenlinien, Specola Vaticana, 1972; Peter, H.; Scheller H.; Atlas für Gitterspekrographen 2250 bis $6500 \AA$ A, Carl Zeiss: Jena, 1982. 
7. Harrison, G.R.; M.I.T. Wave-Length Tables of 100.000 Spectrum Lines, New York, 1939; Meggers, W.E.; Corliss, C.H.; Scribner, B.F.; Tables of Spectraline-Intensities, Nat. Bur. Standards Monograph, 1961, p. 32.

8. IUPAC, "Orange Book", Compendium of Analytical Nomenclature; Freiser, H.; Nancollas, G.H., eds., $2^{\text {nd }}$ ed., Blackwell Scientific Pub.: Oxford, 1987.

9. Hulanicki, A.; IUPAC Commission on General Aspects of Analytical Chemistry, Pure Appl. Chem. 1995, 67,1905.

10. Harvey, C.E.; A Method of Semi-Quantitative Spectrographic Analysis, Glendale, A.R.L., California, 1947.

11. Ramendik, G.I.; Fresenius'J. Anal. Chem. 1990, 337, 772.

12. Sherman, J.; Spectrochim. Acta 1955, 7, 283; Fei He, Van Espen, P.J.; Anal. Chem. 1991, 63, 2237.

13. Danzer, K.; Fresenius'J. Anal. Chem. 1990, 337, 794.

14. Currie, L.A.; Svehla, G.; IUPAC Commission on Analytical Nomenclature; Pure Appl. Chem. 1994, 66, 595.

15. Harter, H.L.; Int. Statist. Rev. 1974, 42, 147; ibid, 1975, 43, 269.

16. Currie. L.A.; Anal. Chem. 1968, 40, 596

17. Kaiser. H.; Fresenius' J. Anal. Chem. 1965, 209, 10; ibid, 1966, 216, 80.

18. Currie, L.A., ed.; Detection in Analytical Chemistry: Importance, Theory, and Practice, ACS Symp. Ser. 361, Washington, 1988, ch. 1.

19. Currie, L.A.; Horwitz, W.; Analusis 1994, 22, 24M.

20. Garden, J.S.; Mitchell, D.G.; Mills, W.N.; Anal. Chem. 1980, 52, 2310.

21. Draper, N.R.; Smith, H.; Applied Regression Analysis, $2^{\text {nd }}$ ed., Wiley: New York, 1981.
22. Wald, A.; Ann. Math. Stat. 1940, 11, 284.

23. Malinowski, E.R.; Howery, D.G.; Factor Analysis in Chemistry, Wiley: New York, 1980.

24. Flury, B.; Common Principal Components and Related Multivariate Models, Wiley: New York, 1988.

25. Martens, H.; Naes, T.; Multivariate Calibration, Wiley: New York, 1989.

26. Natrella, M.G.; Experimental Statistics, National Bureau of Standards Handbook 91: Washington D. C., 1963.

27. MacTaggart, D.L.; Farwell, S.O.; J. Assoc. Off. Anal. Chem. 1992, 75, 594; 614.

28. Danzer, K.; Wagner, M.; Fischbacher, C.; Fresenius' J. Anal. Chem. 1995, $352,407$.

29. Fuller, W.A.; Measurement Error Models, Wiley: New York, 1987.

30. Meloun, M.; Militiký. J.; Forina , M.; Chemometrics for Analytical Chemistry, Ellis Horwood: New York, 1994, vol. 2.

31. Mandel, J.; J. Qual. Technol. 1984, 16, 407.

32. Mandel, J.; The Statistical Analysis of Experimental Data, Wiley: New York, 1964.

33. Hartley, H.O.; Biometrika 1950, 37, 308.

34. Bartlett, M.S.; Proc. Roy. Soc. A 1937, 160, 268.

35. Rousseeuw, P.J.; Lerov, A.M.; Robust Regression and Outlier Detection, Wiley: New York, 1987.

36. Huber, P.J.; Robust Statistics, Wiley: New York, 1981.

37. Sharaf, M.A.; Illman, D.L.; Kowalski, B.R.; Chemometrics, Wiley: New York, 1986. 